\title{
Endodontic Treatment and Restoration of a Mandibular First Premolar Type IV Wiene's Root Canal Configuration with IPS E.Max CAD Technology: A Case Report
}

Talabani RM* ${ }^{*}$, Rassam $\mathbf{R}^{2}$ and Omer $\mathrm{JO}^{3}$

${ }^{1}$ Department of Conservative Dentistry, College of Dentistry/ University of Sulaimani, Iraq

${ }^{2}$ Specialist of Esthetic Dentistry and Implantology in B\&R Dental Center, Iraq

${ }^{3}$ Digital Designer in Fixed Prosthodontic CAD/CAM

Technology in B\&R Dental Center, Iraq

*Corresponding author: Talabani RM, Department of Conservative Dentistry, College of Dentistry/ University of Sulaimani, Kurdistan Region, Sulaimani-Dream Land B-25, Iraq

Received: March 28, 2018; Accepted: April 04, 2018; Published: April 24, 2018

\begin{abstract}
This case report describes an endodontic treatment of a mandibular first premolar with type IV root canal. A 28-year-old male patient reported pain in left mandibular first premolar. Clinical examination showed a large carious lesion with pulp exposure. Radiograph showed no periapical change with intact periodontal ligament space. Mandibular first premolars usually have one canal. The mandibular first premolar may present large number of anatomic vibrations. The clinician should be aware of the configuration of the pulp system. This case presents the diagnosis and clinical management of a mandibular first premolar with two distinct canals in the apical third of root (Type IV Wiene's canal configuration), drawing particular attention to tactile examination of all canal walls and preparation with wave.one gold reciprocating system then obturating it with single cone and AH Plus sealer and finally restored with fiber post and IPS E.Max Cad/Cam technology.
\end{abstract} CAM

Keywords: Mandibular first premolar; Canal configuration; Fiber post; CAD/

\section{Introduction}

Knowledge of the complexity of root canal anatomy and common variants is necessary for the success of root canal treatment [1].

Clinically, it is important to know that a root has a single root canal at the pulp chamber floor that splits into multiple root canals at a certain point of the root canal length; it is also important to determine the level of the root canal where the separation occurs. Root canal spitting in the middle and apical root canal sections may go unnoticed, is more difficult to manage and may affect the cyclic fatigue of endodontic instruments [2].

Weine's classification system of root canal configuration has long history of use and classified the root canals into four types [3]. Type I is one continuous root canal with one orifice and one exit. Type II is a canal with two orifices which combines into one before reaching the portal of exit. Type III refers to two distinct canals which has two distinct orifices and two distinct portal of exit. Type IV refers to canal which has one orifice and diverges into two canals which has separate portal of exit.

The main objective of root canal therapy is thorough shaping and cleaning of all pulp spaces and its complete obturation with an inert filling material. The presence of an untreated canal may be a reason for failure. A canal may be left untreated because the dentist fails to recognize its presence [4].

The mandibular premolars are difficult to treat as they have a high flare-up and failure rate. It may be due to the extreme variations in root canal morphology. Normally the root canal system of the mandibular second premolar is wider buccolingually than mesiodistally with two pulp horns. At the cervical line the root and canal are oval; this shape tends to become round as the canal approaches the middle of the root. If two canals are present, they tend to be round from the pulp chamber to their foramen. Another anatomic variation is that a single, broad root canal may bifurcate into two separate root canals. Direct access to the buccal canal is usually possible, whereas the lingual canal may be very difficult to find. The lingual canal tends to diverge from the main canal at a sharp angle. In addition, the lingual inclination of the crown tends to direct files buccally, making location of a lingual canal orifice more difficult [5].

After root canal treatment, the teeth become weakened due to structural loss that results from canal preparation or extensive previous restoration. That is why they require specialized restoration materials. The use of intracanal posts, especially fibre posts, promotes both the retention of the final restoration and the strengthening of the remaining tooth structure the elasticity of the fibre post is very similar to dentine, and because of this, prevents vertical fracture risk by distributing the stress from the canal walls [6].

Endodontically treated tooth mainly weakened due to dental caries, trauma, or pre-existing restorations. Tooth fracture usually occurs when the root filled tooth is not immediately or properly restored, success rate in endodontically treated tooth with immediate permanent restorations is higher than those with longterm provisional restorations, especially in the posterior teeth with excessive loss of tooth structure [7].

Computer aided design/computer aided manufacturing (CAD/ CAM) systems are widely used in dentistry. CAD/CAM ceramic materials are manufactured under optimized conditions, which can minimize the voids and volume defects. Among the materials used in all-ceramic restorations, lithium disilicate glass ceramic (IPS e.max 


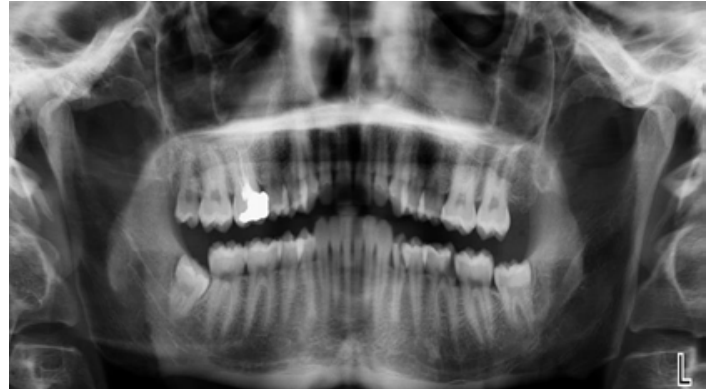

Figure 1: Digital panoramic radiograph showing left mandibular first premolar with large carious lesion.

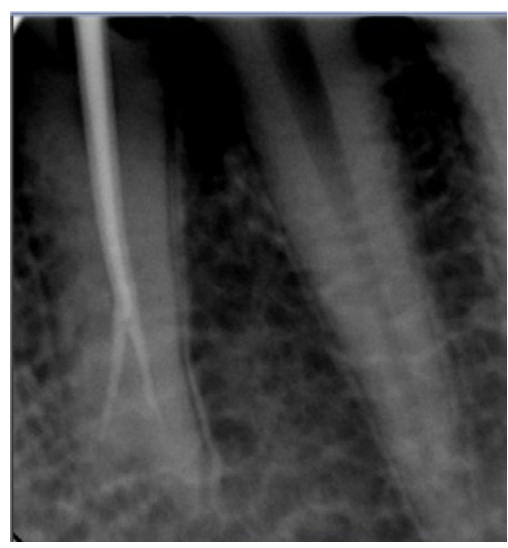

Figure 2: Periapical radiograph showing the working length.

CAD, IvoclarVivadent) not only exhibits a favorable translucency and shade variety, but also has a greater flexural strength [8].

This case report describes the successful diagnosis and treatment of mandibular first premolar with a Type IV Weine's configuration.

\section{Case Presentation}

A 28-year-old male with a noncontributory medical history sought treatment at B\&R private Dental Center. Chief complaint was "pain at night". Clinical examination showed a large class II carious lesion with pulp exposure. The tooth was sensitive to cold testing. Investigation for swelling, sinus tract, and periodontal involvement were negative; the pulp was diagnosed as symptomatic irreversible pulpitis. Preoperative panoramic radiograph revealed no any periapical change and intact periodontal ligament space (Figure 1).

Anesthesia was obtained and an access cavity was prepared. In the floor of pulp chamber only single orifice was detected. Even with the exploration of the access cavity with illumination Loup no other orifices were found. Using K-file size number 10 (Dentsply, Maillefer, Switzerland) the working length was determined radiographically. Access was further widened and a number-10 file with precurve in the apical third was placed alongside of the first file and radiograph was taken. This radiograph confirmed Type IV Weine's configuration (Figure 2).

The biomechanical preparation was carried out with 5.25\% $\mathrm{NaOCl}$ as the irrigant and canals were prepared with primary and medium wave.one gold reciprocating file (Dentsply, X.SmartiQ ).
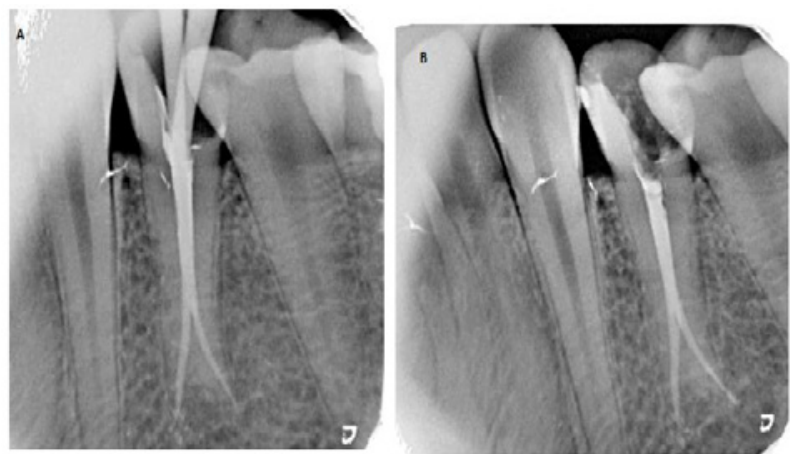

Figure 3: (A) Periapical radiograph showing the master cone (B) Periapical radiograph showing obturated canals.
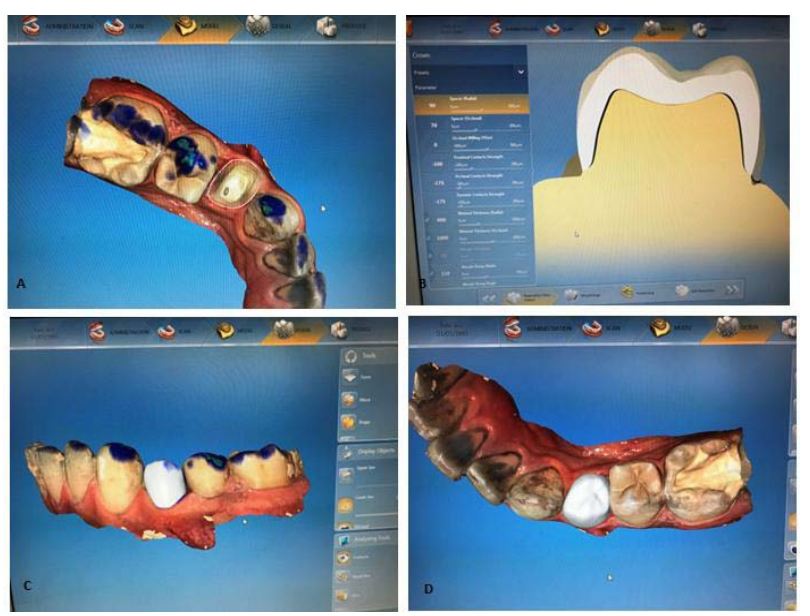

Figure 4: (A) Drawing margins of framework on the virtual model was fabricated with Cerec 3D CAD/CAM (Premium SW 4.5) system (Sirona) (B) Clinical parameters on the virtual model $(C)$ Buccal design of the restoration (D) Occlusal design of the restoration.

The canals were irrigated with saline. The canals were then dried with sterilized paper points, and calcium hydroxide Apexcal (Refill, IvoclarVivadent) dressing was given in the canals was closed with Cavit. Patient was recalled after 10 days. When patient returned, he was totally comfortable. After removal of Cavit, smear layer was removed with 17\% EDTA and canals were irrigated and dried.

Single Cone with AH Plus (DENTSPLY, Switzerland) was used for obturation. The master cone, medium wave.one gold, was inserted to full working length confirmed radiographically and apical tug-back was checked clinically (Figure 3).

For restoration of endodontically treated tooth, the post space was prepared 1 week after the endodontic treatment was completed. The post space was created by removing approximately $5 \mathrm{~mm}$ of endodontic filling material using a thin straight fissure bur. The prepared post space was then cleaned with saline, air dried and acid etched with $37 \%$ phosphoric acid for 15 seconds. This space was rinsed and air dried with oil-free compressed air. A light-cured bonding agent was brushed on the etched surface and uniformly dispersed by a compressed air blast. It was then light cured with for 20 seconds. RelyX" Fiber Post (3M ESPE) was then cured for 20 seconds in order to gain rigidity, before insertion into the post space. Dual- 


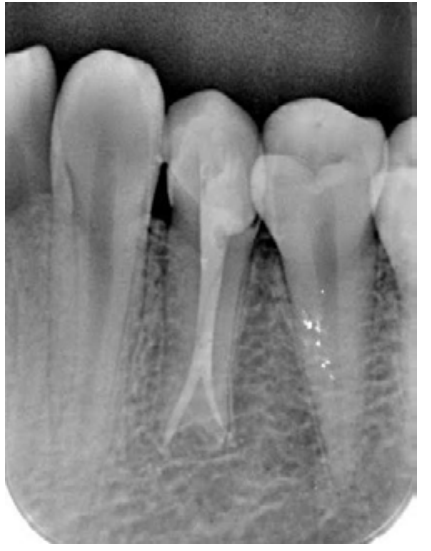

Figure 5: Final restoration.

Cure Core $\bullet \mathrm{X}^{\mathrm{w}}$ (DENTSPLY) flow Build-up Material and Cement for Endodontic Posts was then inserted into the canal chamber after which the blue color RelyX" Fiber Post was inserted. The fiber post and composite were then cured with together for 60 seconds. The coronal enamel was then etched for 20 seconds, rinsed with water and air dried followed by application of bonding agent-which was then light cured. The coronal post was then covered with the Dual-Cure Core $X^{\prime \prime}$ (DENTSPLY) flow for core build up, followed by light curing it for 60 seconds, and finally teeth was prepared with round end tip fissure bur to be restored with IPS E.Max CAD (lithium disilicate glass-ceramic block for the CAD/CAM technique) (Figure 4 and 5).

\section{Discussion}

Variation of root canal configuration, internal and external root anatomy influences the outcome of root canal therapy [9].

The mandibular first premolar is a good example of complex anatomy. It may have extra root, not obvious in preoperative radiograph or fine ribbon-shaped canal system, challenging for shaping, cleaning, and obturation. The study at the University of Washington reported higher failure rate of $11.4 \%$ for nonsurgical root canal treatment of mandibular first premolars [10].

Dou, et al. (2017) [11] reported that $35.96 \%$ of mandibular first premolars have multiple-canal system; however, the actual rate of detecting two canals is relatively low in clinical practice. It is inferred that the additional canal of permanent mandibular first premolars was often missed. The inability to locate, debride and obturate a second canal is often a major reason for failure when employing endodontic treatment for permanent mandibular first premolar.

Albuquerque, et al. (2014) [12] stated that the mandibular first premolars being comparatively twice more likely to present with two canals $(23.55 \%)$ than second premolars (12.64\%).

The coronal and radicular anatomy and landmarks are prerequisites for the detection of variable anatomy for example atypical crown dimensions, additional cusps, tubercles, deep longitudinal developmental grooves, and fusion of teeth all point to anomalous tooth morphology, which could be accompanied by anomalous endodontic morphology [13].

Borkar and de Ataide (2015) [14] observed that to improve the retention and mechanical performance of the restored teeth, placement of glass fiber post was recommended with three important features: the elastic modulus of fiber posts is similar to that of dentin; posts are adhesively luted to the canal, and adhesive bonding to the composite core material which offer more homogenous distribution of stresses than rigid posts and reducing the possibility of vertical root fracture.

In this case, a novel technique of a resin-bonded lithium disilicate with CAD/CAM technology has demonstrated to restore endodontically treated tooth because the lower coefficient of thermal expansion (CTE) of lithium disilicate (Emax, IvoclarVivadent) helpful in better improvement of internal adaptation and marginal integrity also CAD/CAM manufacturing, concerns regarding dimensional change can be ignored as the ceramic ingots were milled at room temperature [15].

\section{Conclusion}

Clinician should be aware of variation related to canal configuration and types in mandibular first premolars. Tactile examination is a key step in locating the extra/split canal. Fiber post and coronal coverage is important to prevent coronal leakage and improve the prognosis of the restoration.

\section{Clinical Relevance}

Mandibular premolars are known to have numerous anatomic variations of their roots and root canals, which are a challenge to treat endodontically. A thorough description of every step of treatment including preoperative diagnosis, intraoperative identification and management for the successful management of these complex cases should be considered.

\section{Acknowledgement}

The authors wish to thanks Dr. Bamo Namiq Abdulkareem (Head of B\&R Dental Center) for his support and assistance.

\section{References}

1. Lee KW, Kim Y, Perinpanayagam H, Lee JK, Yoo YJ, Lim SM, et al. Comparison of alternative image reformatting techniques in micro-computed tomography and tooth clearing for detailed canal morphology. J Endod. 2014; 40: 417-422.

2. Martins JNR, Marques D, Mata A, Caramês J. Root and root cana morphology of the permanent dentition in a Caucasian population: a conebeam computed tomography study. Int J Endod. 2017; 50: 1013-1026.

3. Chang SW, Lee JK, Lee Y, Kum KY. In-depth morphological study of mesiobuccal root canal systems in maxillary first molars: review. Restor Dent Endod. 2013; 38: 2-10.

4. Vertucci FJ. Root canal morphology and its relationship to endodontic procedures. Endodontic Topics. 2005; 10: 3-29.

5. Chalil NM, Kini S, Jose S, Narayanan A, Salahudeen S, Peedikayil FC. Endodontic Treatment of a Mandibular Second Premolar with Type IV Wiene's Root Canal: A Case Report. Case Rep Dent. 2014; 2014: 731467.

6. Bayram HM, Bayram E, Kul E, Göktürk H. Effect of intracanal medicament on bond strength of fibre posts. Biotechnology \& Biotechnological Equipment. 2016; 30: 785-789.

7. Suksaphar W, Banomyong D, Jirathanyanatt $T$, Ngoenwiwatkul Y. Survival rates against fracture of endodontically treated posterior teeth restored with full-coverage crowns or resin composite restorations: a systematic review. Restor Dent Endod. 2017; 42: 157-167. 
8. Yu W, Guo K, Zhang B, Weng W. Fracture resistance of endodontically treated premolars restored with lithium disilicate CAD/CAM crowns or onlays and luted with two luting agents. Dent Mater J. 2014; 33: 349-354.

9. Hosseinpour S, Kharazifard MJ, Khayat A, Naseri M. Root Canal Morphology of Permanent Mandibular Premolars in Iranian Population: A Systematic Review. Iran Endod J. 2016; 11: 150-156.

10. Chourasia HR, Boreak N, Tarrosh MY, Mashyakhy M. Root canal morphology of mandibular first premolars in Saudi Arabian southern region subpopulation. Saudi Endod J. 2017; 7: 77-81.

11. Dou L, Li D, Xu T, Tang Y, Yang D. Root anatomy and canal morphology of mandibular first premolars in a Chinese population. Sci Rep. 2017; 7: 750.
12. Albuquerque D, Kottoor J, Hammo M. Endodontic and clinical considerations in the management of variable anatomy in mandibular premolars: a literature review. Bio Med Research International. 2014; 2014

13. Nallapati S. Three canal mandibular first and second premolars: a treatment approach. A case report. Journal of Endodontics. 2005; 31: 474-476.

14. Borkar S, de Ataide ID. Management of a Massive Resorptive Lesion with Multiple Perforations in a Molar: Case Report. J Endod. 2015; 41: 753-758.

15. Chieh Liu M. Restoration of endodontically treated premolars and molars: A Review of Rationales and Techniques. Journal of Prosthodontics and Implantology. 2014; 3: 2-16
Austin J Dent - Volume 5 Issue 5 - 2018

ISSN : 2381-9189 | www.austinpublishinggroup.com

Talabani et al. (C) All rights are reserved
Citation: Talabani RM, Rassam R and Omer JO. Endodontic Treatment and Restoration of a Mandibular First Premolar Type IV Wiene's Root Canal Configuration with IPS E.Max CAD Technology: A Case Report. Austin J Dent. 2018; 5(5): 1115. 